\title{
Algorithms and Bounds for Dynamic Causal Modeling of Brain Connectivity
}

\author{
Shun Chi Wu, Member, IEEE, and A. Lee Swindlehurst, Fellow, IEEE
}

\begin{abstract}
Recent advances in neurophysiology have led to the development of complex dynamical models that describe the connections and causal interactions between different regions of the brain. These models are able to accurately mimic the event-related potentials observed by EEG/MEG measurement systems, and are considered to be key components for understanding brain functionality. In this paper, we focus on a class of nonlinear dynamic causal models (DCM) that are described by a set of connectivity parameters. In practice, the DCM parameters are inferred using data obtained by an EEG or MEG sensor array in response to a certain event or stimulus, and then used to analyze the strength and direction of the causal interactions between different brain regions. The usefulness of these parameters in this process will depend on how accurately they can be estimated, which in turn will depend on noise, the sampling rate, number of data samples collected, the accuracy of the source localization and reconstruction steps, etc. The goals of this paper are to present several algorithms for DCM parameter estimation, derive Cramér-Rao performance bounds for the estimates, and compare the accuracy of the algorithms against the theoretical performance limits under a variety of circumstances. The influence of noise and sampling rate will be explicitly investigated.
\end{abstract}

Index Terms-Brain connectivity, dynamic causal modeling, EEG, MEG.

\section{INTRODUCTION}

$\mathbf{R}$ ECENT research in understanding brain functionality has focused on describing the connectivity between different cortical regions and how these regions interact when executing certain sensorimotor and cognitive tasks. Two distinct approaches to describing brain connectivity have emerged in the neurological literature. The first, referred to as functional connectivity, endeavors to explain brain interactions through the temporal correlation between spatially remote neurophysiological event-related potentials (ERP's) measured with electroencephalography (EEG) or magnetoencephalography (MEG) systems. Calculation of the functional connectivity normally requires no knowledge about the structure of the neural system of interest, and thus is often considered to be "model-free." However, no causal relationship or directed

Manuscript received April 11, 2012; revised January 19, 2013; accepted March 06, 2013. Date of publication March 27, 2013; date of current version May 10, 2013. The associate editor coordinating the review of this manuscript and approving it for publication was Yufei Huang.

The authors are with the Department of Electrical Engineering and Compute Science, University of California, Irvine, CA 92697 USA (e-mail: scwu@uci. edu; swindle@uci.edu).

Color versions of one or more of the figures in this paper are available online at http://ieeexplore.ieee.org.

Digital Object Identifier 10.1109/TSP.2013.2255040 causal influence of one brain region on another can be inferred from the observed correlations. On the other hand, effective connectivity corresponds to a specific parametric model that represents the simplest brain circuit that would produce the same temporal relationship observed experimentally in EEG/MEG data from different cortical regions. While effective connectivity allows one to establish causal relationships between brain regions, one must postulate a suitable causal model in which the regions of interest are constrained by a combination of neuroanatomical, neuropsychological and functional neuroimaging data [1]-[3]. Both types of brain connectivity analysis offer a rich set of interesting applications in signal processing and systems theory.

Several approaches for effective connectivity analysis have been discussed in the literature [4]-[13]. In structural equation modeling (SEM), path coefficients are used to quantify the influence of directional connections between cortical regions, and are estimated through the minimization of the difference between the modeled and observed covariance matrices of the EEG/MEG data in the regions of interest [4], [7], [12], [13]. SEM models are typically restricted to low complexity cases, and models with reciprocal connections or loops are not identifiable. In particular, SEM does not account for temporal ordering of the signals; for example, if all the time series in a given region are permuted in the same fashion, the estimated path coefficients will not change [4], [13]. In contrast, multivariate autoregressive models assume present measurements are linear combinations of previous values, which explicitly takes into account the causal dependence of the present on the past [5], [8], [11]-[13]. Based on the Granger causality between time series [5], [14], the directional influence of one signal on another can be determined based on multivariate spectral measures like the directed transfer function [5], [8], [11]. In addition, the connections are allowed to be reciprocal and the evaluation of the directions of interactions are possible even without prior knowledge about the network [5], [11]-[13].

Dynamic causal modeling (DCM) for the generation of EEG/MEG ERPs based on the neural mass model of [15] is another well-known approach for inferring cortical connectivity [9], [10], [13], [16]. In DCM, the brain is regarded as a nonlinear dynamical network. Each cortical region in the DCM network is comprised of three subpopulations linked by intrinsic connections, and different regions communicate through extrinsic excitatory forward, backward and lateral connections. The activity of each cortical area is described by unobserved state variables and the output of the model is presumed to be the sources of the EEG/MEG ERPs. In this paper, we will focus on the DCM approach for modeling effective brain connectivity. 
While the EEG and MEG data used for DCM provide direct measurements of brain activity at millisecond resolutions (the time scale on which many brain events unfold), obtaining good spatial resolution requires large sensor arrays, accurate forward models for signal propagation and special localization methods [17]-[21]. Furthermore, the DCM parameters are not observed directly in the EEG/MEG data, but must be estimated based on signals reconstructed from each of the relevant cortical regions [10], [22], typically via Bayesian inference (e.g., maximum a posteriori or MAP estimation). MAP approaches based on direct multi-dimensional optimization or using iterative local linear approximations of the state equations have been proposed in [6], [10], although either details of the implementation are missing or numerical rather than exact approaches are used to find derivatives of the system outputs with respect to the model parameters. In situations where no prior information is available about the parameters, the maximum likelihood (ML) approach can be used instead. In either case, the resulting algorithms are very complicated and computationally intensive, since DCM is based on a multivariable nonlinear differential equation.

The goal of this paper is to discuss dynamic causal modeling for brain connectivity from an estimation theoretic viewpoint. After presenting the DCM approach for EEG/MEG ERPs based on a neural mass model in Section II, in Section III we develop a method for obtaining the Cramér-Rao bound (CRB) [23] and the posterior CRB [24] for the DCM parameters. This is a non-trivial exercise, since the observations result from a nonlinear dynamical system that depends on the parameters in a complicated way. In particular, calculation of the CRB requires evaluation of the Fisher (or, more generally, posterior) information matrix, which involves derivatives of the conditional probability distribution function (pdf) of the observations with respect to the parameters. The nonlinear differential equation at the heart of DCM does not admit an analytical description of the effect of the model parameters on the output, so standard methods cannot be used. Instead, we calculate the CRB and posterior CRB using the "derivative system" approach in [25] appropriately modified to handle the availability of a priori parameter distributions.

In Section IV, we describe methods for implementing MAP and $\mathrm{ML}$ approaches for DCM parameter estimation. For the same reasons listed above, application of MAP and ML estimation to the DCM problem is also non-trivial and computationally intensive, involving repeated execution of the corresponding DCM derivative system as the parameter estimates are updated. While results based on MAP estimation for DCM have appeared elsewhere [10], details of the how the algorithms are actually implemented have not. In addition to the MAP and ML algorithms, in Section IV we also present an alternative approach based on the so-called dual extended Kalman filter (dEKF) [26]. Since the dEKF is based on a local linearization of the model at each time step, it as not accurate as MAP and ML for low sample rates, but it is simpler to implement since it can simultaneously estimate the DCM state and model parameters at each time sample, without resorting to use of the derivative system.

From a neurophysiological point of view, one of the most important outcomes of the DCM estimation process is information about the existence and strength of the forward or backward connections between different brain regions, since this provides clues to the causal interrelationships between these regions. In Section V, we pose the detection of forward and backward connections as a model-order selection problem, and discuss methods for determining the presence or absence of such connections. Finally, in Section VI, we conduct a series of DCM simulation experiments to study the performance of the dEKF, ML and MAP algorithms as a function of changes in the noise environment, sampling rate and sample size compared with the derived Cramér-Rao bounds. The MAP and ML algorithms are shown to provide performance very close to their respective bounds, while the performance of dEKF only approaches the bound as the sample rate is increased. We also use simulations to study the ability of the algorithms to correctly determine the underlying connectivity between the modeled brain regions.

\section{Dynamic Causal Modeling For ERP Generation}

DCM is used to model the generation of ERPs observed by EEG/MEG recordings. It is based the neural mass model (NMM) of [15], in which the causal interactions between different regions of the brain are described by a set of links and connectivity parameters. In this section, we provide a brief physical description of the mathematics underlying the model used in the DCM approach. Additional details can be found in [9], [10], [15].

Each cortical region in the DCM network is assumed to be comprised of three neuronal subpopulations referred to as spiny stellate cells, inhibitory interneurons and pyramidal cells. These subgroups are linked by intrinsic connections $\gamma_{1}, \ldots, \gamma_{4}$, while different regions communicate through extrinsic excitatory forward, backward and lateral connections. Fig. 1 shows a block diagram that illustrates the DCM structure for a given brain region $i$, showing the three neuronal subregions and the various inputs, outputs and internal connections. Normally, extrinsic inputs $u_{i}(t)$ that are due to stimuli (audio, visual, etc) external to the brain act directly on the spiny stellate cells, which also receive signals $y_{s}^{(j)}(t)$ from the other cortical region through both forward and lateral connections. Pyramidal cells and inhibitory interneurons also receive signals from the other cortical region, although these are via backward and lateral connections. Region $i$ 's output, $y_{s}^{(i)}(t)$, is a mixture of potentials induced by excitatory and inhibitory currents, and is generated in the pyramidal cells [9], [10]. The signal $y_{s}^{(i)}(t)$ is presumed to represent the EEG/MEG ERP for region $i$.

According to the dynamic model of [9], [10], [15], the activity of the $i$ th cortical region is described by an eight-element state vector $\mathbf{x}^{(i)}(t)=\left[x_{1}^{(i)}(t) \ldots x_{8}^{(i)}(t)\right]^{T}$. As shown in Fig. 1, each state variable is associated with one of the three neural subpopulations in a given region. The evolution of the state vector $\mathbf{x}^{(i)}(t)$ is assumed to be governed by the following nonlinear differential equation:

$$
\begin{aligned}
\dot{\mathbf{x}}^{(i)}(t) & =\mathbf{f}^{(i)}\left(t, \mathbf{x}^{(i)}, u_{i}\right) \\
y_{s}^{(i)}(t) & =x_{2}^{(i)}(t)-x_{3}^{(i)}(t),
\end{aligned}
$$




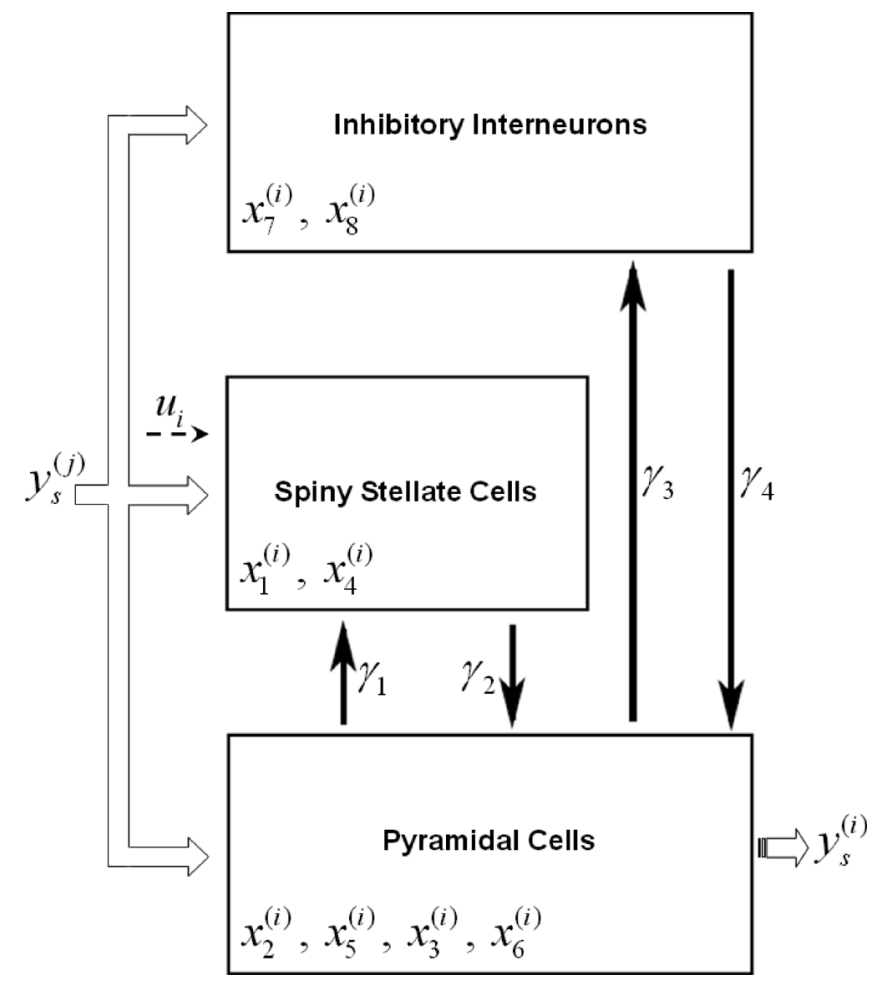

Fig. 1. Single cortical region in DCM. Each cortical region in the DCM network is comprised of three subpopulations linked by intrinsic connections, and different regions communicate through extrinsic excitatory forward,backward and lateral connections.

where the elements of the vector $\mathbf{f}^{(i)}\left(t, \mathbf{x}^{(i)}, u_{i}\right)=$ $\left[f_{1}^{(i)}(t) \ldots f_{8}^{(i)}(t)\right]^{T}$ are specified as

$$
\begin{aligned}
f_{1}^{(i)}(t)= & x_{4}^{(i)}(t), \\
f_{2}^{(i)}(t)= & x_{5}^{(i)}(t), \\
f_{3}^{(i)}(t)= & x_{6}^{(i)}(t), \\
f_{4}^{(i)}(t)= & \frac{H_{e}}{\tau_{e}}\left\{\sum_{j=1 ; j \neq i}^{m} a_{i j}^{F} S\left(y_{s}^{(j)}\left(t-\delta_{i j}\right)\right)\right. \\
& +\sum_{j=1 ; j \neq i}^{m} a_{i j}^{L} S\left(y_{s}^{(j)}\left(t-\delta_{i j}\right)\right)+c_{i} u_{i}(t) \\
& \left.+\gamma_{1} S\left(y_{s}^{(i)}(t)\right)\right\}-\frac{2}{\tau_{e}} x_{4}^{(i)}(t)-\frac{x_{1}^{(i)}(t)}{\tau_{e}^{2}}, \\
f_{5}^{(i)}(t)= & \frac{H_{e}}{\tau_{e}}\left\{\sum_{j=1 ; j \neq i}^{m} a_{i j}^{B} S\left(y_{s}^{(j)}\left(t-\delta_{i j}\right)\right)\right. \\
& +\sum_{j=1 ; j \neq i}^{m} a_{i j}^{L} S\left(y_{s}^{(j)}\left(t-\delta_{i j}\right)\right) \\
& \left.+\gamma_{2} S\left(x_{1}^{(i)}(t)\right)\right\}-\frac{2}{\tau_{e}} x_{5}^{(i)}(t)-\frac{x_{2}^{(i)}(t)}{\tau_{e}^{2}}, \\
f_{6}^{(i)}(t)= & \frac{H_{i}}{\tau_{i}} \gamma_{4} S\left(x_{7}^{(i)}(t)\right)-\frac{2}{\tau_{i}} x_{6}^{(i)}(t)-\frac{x_{3}^{(i)}(t)}{\tau_{i}^{2}}, \\
f_{7}^{(i)}(t)= & x_{8}^{(i)}(t),{ }_{m}^{m} a_{i j}^{B} S\left(y_{s}^{(j)}\left(t-\delta_{i j}\right)\right) \\
f_{8}^{(i)}(t)= & \frac{H_{e}}{\tau_{e}}\left\{\sum_{j=1 ; j \neq i} \sum^{m}\right)
\end{aligned}
$$

$$
\begin{aligned}
& +\sum_{j=1 ; j \neq i}^{m} a_{i j}^{L} S\left(y_{s}^{(j)}\left(t-\delta_{i j}\right)\right) \\
& \left.+\gamma_{3} S\left(y_{s}^{(i)}(t)\right)\right\}-\frac{2}{\tau_{e}} x_{8}^{(i)}(t)-\frac{x_{7}^{(i)}(t)}{\tau_{e}^{2}} .
\end{aligned}
$$

The parameters of the differential equation are explained below. The constant $c_{i}$ represents the strength of the influence of the input excitation $u_{i}(t)$, which may not be present in all modeled brain regions. Parameters $a_{i j}^{F}, a_{i j}^{B}, a_{i j}^{L}$ define the connectivity of the extrinsic forward, backward, and lateral connections between regions $i$ and $j$, respectively, while $\delta_{i j}$ represents the conduction delay between these two regions. The coupling parameters $\gamma_{1}, \ldots, \gamma_{4}$ control the strength of the intrinsic connections between the three subpopulations, and are proportional to the average number of synapses between the pyramidal cells and the excitatory and inhibitory feedback elements. The remaining constants are relevant to two transformations on which the system dynamics are based. The first transformation is linear and converts the average pulse density of the action potentials arriving at the population of neurons into an average postsynaptic membrane potential. In particular, $H_{e}$ and $H_{i}$ control the maximum postsynaptic potentials, and $\tau_{e}$ and $\tau_{i}$ represent the lumped time constants of the passive membrane and all other spatially distributed delays in the dendritic network. The subscripts $e$ and $i$ are used to indicate whether these potentials are either excitatory and inhibitory, respectively. The second transformation $S(v)$ is a nonlinear sigmoid function that converts the average membrane potential of the given population into an average firing rate for the action potentials, and is given by [9]

$$
S(v)=\frac{2 e_{0}}{1+e^{(-r v)}}-e_{0},
$$

where $e_{0}$ determines the maximum firing rate of the neural population and $r$ controls the steepness of the sigmoidal transformation.

The state equation parameters can be divided into two groups: (1) intrinsic parameters $\left(H_{e}, H_{i}, \tau_{e}, \tau_{i}, \gamma_{1}, \gamma_{2}, \gamma_{3}, \gamma_{4}, e_{0}, r\right)$ that control the dynamics within a cortical region and are normally fixed based on existing knowledge about the region [15], and (2) extrinsic parameters $\left(a_{i j}^{F}, a_{i j}^{B}, a_{i j}^{L}, \delta_{i j}, c_{i}\right)$ that specify the directed causal interactions among different cortical regions and that are the parameters for which inference is required. The extrinsic parameters are always positive since they represent the strength of a particular cortical connection; the causal direction of the connection is specified by the name of the extrinsic parameter itself (forward, backward or lateral) [9], [10], [16]. While the values of the extrinsic parameters are unknown and must be estimated, a priori information is often available for them in the form of a prior distribution. Since the parameters are positive, these prior pdfs are typically chosen to be lognormal.

For a general DCM network composed of $m$ interconnected brain regions, a network-level model is obtained by combining together all of the equations for each region into a single nonlinear differential equation with $8 m$ states:

$$
\begin{aligned}
\dot{\mathbf{x}}(t) & =\mathbf{f}(t, \mathbf{x}, \mathbf{u}) \\
\mathbf{y}(t) & =\mathbf{C x}(t)+\mathbf{w}(t)=\mathbf{y}_{s}(t)+\mathbf{w}(t),
\end{aligned}
$$


where $\mathbf{x}(t)=\left[\left(\mathbf{x}^{(1)}(t)\right)^{T} \ldots\left(\mathbf{x}^{(m)}(t)\right)^{T}\right]^{T}$ and $\mathbf{f}(t, \mathbf{x}, \mathbf{u})=$ $\left[\mathbf{f}^{(1)}\left(t, \mathbf{x}, u_{1}\right)^{T} \ldots \mathbf{f}^{(m)}\left(t, \mathbf{x}, u_{m}\right)^{T}\right]^{T}$ represent the network state vector and its derivatives, $\mathbf{u} \in \mathbb{R}^{m \times 1}$ contains the external inputs to each region (some of which may be zero), and $\mathbf{y}(t) \in \mathbb{R}^{m \times 1}$ represents the measured ERPs for each region in the presence of measurement noise $\mathbf{w}(t) \in \mathbb{R}^{m \times 1}$. In deriving the CRB as well as the MAP and ML algorithms, we will assume that $\mathbf{w}(t)$ is zero-mean Gaussian with independent elements of equal variance $\sigma^{2}$. The noise free ERPs are represented as $\mathbf{y}_{s}(t)=\mathbf{C x}(t)=\left[y_{s}^{(1)}(t) \ldots y_{s}^{(m)}(t)\right]^{T} \in \mathbb{R}^{m \times 1}$, where $\mathbf{C} \in \mathbb{R}^{m \times 8 m}$ is a constant matrix with nonzero elements only in the following locations:

$$
\begin{aligned}
& C_{i, 8(i-1)+2}=1, \\
& C_{i, 8(i-1)+3}=-1,
\end{aligned}
$$

for $i=1, \ldots, m$.

Associated with the nonlinear state equations in (3) are a set of parameters represented by the vector $\boldsymbol{\theta}$. This vector contains the unknown extrinsic parameters $a_{i j}^{F}, a_{i j}^{B}, a_{i j}^{L}, \delta_{i j}, c_{i}$ for each cortical region $i$ and every region $j$ to which $i$ is connected. In the sections that follow, we discuss algorithms for estimating $\boldsymbol{\theta}$ from the measurements $\mathbf{y}(t)$, lower bounds on the estimation performance, and methods for determining which types of connections exist between different regions.

\section{POSTERIOR CRB AND CRB FOR ESTIMATION IN NONLINEAR SYSTEMS}

The posterior CRB [23], [24] is appropriate for establishing estimation performance bounds for problems where a priori distributions for the parameters of interest are available. Let $\mathbf{Y}=\left[\mathbf{y}\left(t_{1}\right) \ldots \mathbf{y}\left(t_{N}\right)\right] \in \mathbb{R}^{m \times N}$ represent a matrix containing the ERPs for each of the $m$ regions over $N$ time samples. Assume the parameter vector $\boldsymbol{\theta}$ has $n$ elements and denote the joint pdf of the data and parameters as $p(\mathbf{Y}, \boldsymbol{\theta})$. According to the posterior $\mathrm{CRB}$ (PCRB), a lower bound for the mean-squared error (MSE) of an estimator $\hat{\boldsymbol{\theta}}=\mathbf{q}(\mathbf{Y})$ is given by

$$
\mathbf{V} \equiv E\left\{[\mathbf{q}(\mathbf{Y})-\boldsymbol{\theta}][\mathbf{q}(\mathbf{Y})-\boldsymbol{\theta}]^{T}\right\} \geq \mathbf{J}^{-1},
$$

where the posterior information matrix $\mathbf{J}$ is $n \times n$ with elements

$$
\mathbf{J}_{i j}=E\left\{-\frac{\partial^{2} \ln p(\mathbf{Y}, \boldsymbol{\theta})}{\partial \theta_{i} \partial \theta_{j}}\right\} \quad i, j=1, \ldots, n .
$$

Since $p(\mathbf{Y}, \boldsymbol{\theta})=p(\mathbf{Y} \mid \boldsymbol{\theta}) p(\boldsymbol{\theta}), \mathbf{J}_{i j}$ can be decomposed into two parts:

$$
\begin{aligned}
\mathbf{J}_{i j} & =E\left\{-\frac{\partial^{2} \ln p(\mathbf{Y} \mid \boldsymbol{\theta})}{\partial \theta_{i} \partial \theta_{j}}\right\}+E\left\{-\frac{\partial^{2} \ln p(\boldsymbol{\theta})}{\partial \theta_{i} \partial \theta_{j}}\right\} \\
& =\mathbf{J}_{D, i j}+\mathbf{J}_{P, i j}
\end{aligned}
$$

where $\mathbf{J}_{D, i j}$ and $\mathbf{J}_{P, i j}$ represent the elements of the Fisher information matrix (FIM) and a priori information matrix (AIM), respectively. Note that when a priori distributions of the parameters are noninformative or unavailable, only the FIM contributes to $\mathbf{J}$ and the lower bound for the MSE of (4) becomes the standard CRB rather than the posterior CRB.
Calculation of the AIM in (6) for the DCM application is straightforward, but the FIM involves derivatives of a function of the nonlinear system outputs with respect to the parameters. Since an analytical expression for the DCM system output in terms of the parameters does not exist, these derivatives cannot be evaluated directly. Instead, we resort to the approach of [25], which shows that the FIM for a data set generated by a nonlinear system with additive Gaussian measurement noise can be expressed in terms of the outputs of its derivative system, which is itself also described by a set of nonlinear differential equations. In the discussion that follows, we define the DCM derivative system and show how to compute the FIM from its output.

The derivative system for the DCM model in (3) is given by

$$
\begin{aligned}
\dot{\mathcal{X}}(t) & =\mathcal{F}(t) \\
\mathcal{Y}_{\boldsymbol{\theta}}(t) & =\mathcal{C} \mathcal{X}(t),
\end{aligned}
$$

for $t \geq t_{0}$, where

$$
\begin{aligned}
& \mathcal{X}(t)= {\left[\mathbf{x}^{T}(t) \frac{\partial \mathbf{x}^{T}(t)}{\partial \theta_{1}} \ldots \frac{\partial \mathbf{x}^{T}(t)}{\partial \theta_{n}}\right]^{T} \in \mathbb{R}^{8 m(n+1) \times 1} } \\
& \mathcal{F}(t)= {\left[\begin{array}{c}
\mathbf{f}(t, \mathbf{x}, \mathbf{u}) \\
\frac{\partial \mathbf{f}(t, \mathbf{x}, \mathbf{u})}{\partial \theta_{1}}+\mathbf{M}_{J}(t) \frac{\partial \mathbf{x}(t)}{\partial \theta_{1}} \\
\vdots \\
\frac{\partial \mathbf{f}(t, \mathbf{x}, \mathbf{u})}{\partial \theta_{n}}+\mathbf{M}_{J}(t) \frac{\partial \mathbf{x}(t)}{\partial \theta_{n}}
\end{array}\right] \in \mathbb{R}^{8 m(n+1) \times 1} } \\
& \mathcal{Y}_{\boldsymbol{\theta}}(t)=\left[\begin{array}{c}
\frac{\partial y^{(1)}(t)}{\partial \boldsymbol{\theta}} \\
\vdots \\
\frac{\partial y^{(m)}(t)}{\partial \boldsymbol{\theta}}
\end{array}\right]=\left[\begin{array}{c}
\frac{\partial y_{s}^{(1)}(t)}{\partial \boldsymbol{\theta}} \\
\vdots \\
\frac{\partial y_{s}^{(m)}(t)}{\partial \boldsymbol{\theta}}
\end{array}\right] \\
&=\left[\begin{array}{c}
\frac{\partial\left(x_{2}^{(1)}(t)-x_{3}^{(1)}(t)\right)}{\partial \boldsymbol{\theta}} \\
\vdots \\
\frac{\partial\left(x_{2}^{(m)}(t)-x_{3}^{(m)}(t)\right)}{\partial \boldsymbol{\theta}}
\end{array}\right] \in \mathbb{R}^{m n \times 1} .
\end{aligned}
$$

The matrix $\mathcal{C}$ is defined implicitly from the expression for $\mathcal{Y}_{\boldsymbol{\theta}}(t)$ and $\mathbf{M}_{J}(t) \in \mathbb{R}^{8 m \times 8 m}$ is the Jacobian matrix of $\mathbf{f}(t)$ with respect to $\mathbf{x}$ :

$$
\mathbf{M}_{J}(t)=\left[\left(\frac{\partial \mathbf{f}^{(1)}(t)}{\partial \mathbf{x}}\right)^{T} \cdots\left(\frac{\partial \mathbf{f}^{(m)}(t)}{\partial \mathbf{x}}\right)^{T}\right]^{T}
$$

where

$$
\begin{aligned}
& \frac{\partial \mathbf{f}^{(i)}(t)}{\partial \mathbf{x}(t)} \in \mathbb{R}^{8 \times 8 m} \\
& =\left[\begin{array}{ccccccc}
\frac{\partial f_{1}^{(i)}(t)}{\partial x_{1}^{(1)}} & \cdots & \frac{\partial f_{1}^{(i)}(t)}{\partial x_{8}^{(1)}} & \ldots & \frac{\partial f_{1}^{(i)}(t)}{\partial x_{1}^{(m)}} & \ldots & \frac{\partial f_{1}^{(i)}(t)}{\partial x_{8}^{(m)}} \\
\vdots & \ddots & \vdots & \ddots & \vdots & \ddots & \vdots \\
\frac{\partial f_{8}^{(i)}(t)}{\partial x_{1}^{(1)}} & \cdots & \frac{\partial f_{8}^{(i)}(t)}{\partial x_{8}^{(1)}} & \cdots & \frac{\partial f_{8}^{(i)}(t)}{\partial x_{1}^{(m)}} & \ldots & \frac{\partial f_{8}^{(i)}(t)}{\partial x_{8}^{(m)}}
\end{array}\right],
\end{aligned}
$$

and where we have dropped the notation for the explicit dependence of $\mathbf{f}^{(i)}(t)$ on $\mathbf{x}, \mathbf{u}$.

Expressions for the above derivatives are provided in the appendix. Once the derivative system is defined and initialized, standard numerical integration methods can be used to generate 
samples of the output, and the Fisher information matrix can then be obtained through

$$
\mathbf{J}_{D}=\frac{1}{\sigma^{2}} \sum_{j=1}^{N} \sum_{i=1}^{m} \mathbf{P}_{i} \mathcal{Y}_{\boldsymbol{\theta}}\left(t_{j}\right) \mathcal{Y}_{\boldsymbol{\theta}}^{T}\left(t_{j}\right) \mathbf{P}_{i}^{T} \in \mathbb{R}^{n \times n},
$$

where $\mathbf{P}_{i} \in \mathbb{R}^{n \times m n}$ is defined as

$$
\mathbf{P}_{i}=\left[\mathbf{0}^{(1)} \cdots \mathbf{0}^{(i-1)} \mathbf{I}_{n} \mathbf{0}^{(i+1)} \cdots \mathbf{0}^{(m)}\right],
$$

with $\mathbf{0}^{(i)}$ representing an $n \times n$ zero matrix and $\mathbf{I}_{n}$ an $n \times n$ identity matrix. Both the DCM and its derivative system are typically initialized with all state variables set to zero, since no ERP is assumed to be generated without a stimulus. The performance of the algorithms described in Section IV will be compared against the CRB and PCRB derived above.

\section{DCM PARAMETER ESTIMATION}

As with the CRB, the fact that the likelihood function cannot be explicitly expressed as a function of the parameter vector $\boldsymbol{\theta}$ is also a difficulty for ML and MAP estimation algorithms. To address this issue, a local linear approximation to the likelihood function can be used to derive iterative algorithms for finding approximate solutions to the ML and MAP estimation problems. While results based on MAP estimation for the DCM applied to fMRI data have appeared in [6], details of how the algorithms are actually implemented have not been given, and the performance of ML and MAP estimators for the EEG/MEG ERP model has not been studied. Moreover, [6] resorts to the use of numerical approaches for calculation of the local gradient matrix that are not derived and explained only qualitatively. In what follows, we provide a more complete mathematical description of how the ML and MAP algorithms are applied to estimating the parameters of the nonlinear DCM system.

\section{A. Likelihood Function and ML Estimator}

We begin with the simpler case where no a priori distributions are assumed for the DCM parameters. Given the observation model in (3), the likelihood function is calculated via a local linear approximation of $\mathbf{y}_{s}(t)$ in terms of the current estimates of the parameters $\hat{\boldsymbol{\theta}}_{i-1}$ in [6], [10]:

$$
\mathbf{y}_{s}(t ; \boldsymbol{\theta}) \approx \mathbf{y}_{s}\left(t ; \hat{\boldsymbol{\theta}}_{i-1}\right)+\mathbf{J}_{\hat{\boldsymbol{\theta}}_{i-1}}(t)\left(\boldsymbol{\theta}-\hat{\boldsymbol{\theta}}_{i-1}\right)
$$

where

$$
\mathbf{J}_{\hat{\boldsymbol{\theta}}_{i-1}}(t)=\left[\begin{array}{c}
{\left[\frac{\partial y_{s}^{(1)}(t)}{\partial \boldsymbol{\theta}}\right]_{\hat{\boldsymbol{\theta}}_{i-1}}^{T}} \\
\vdots \\
{\left[\frac{\partial y_{s}^{(m)}(t)}{\partial \boldsymbol{\theta}}\right]_{\hat{\boldsymbol{\theta}}_{i-1}}^{T}}
\end{array}\right] \in \mathbb{R}^{m \times n} .
$$

As described below, evaluation of the local gradient matrix $\mathbf{J}_{\hat{\boldsymbol{\theta}}_{i-1}}(t)$ plays an important role in the parameter estimation. In [6], $\mathbf{J}_{\hat{\boldsymbol{\theta}}_{i-1}}(t)$ is evaluated using a two-step approach, in which the DCM outputs are evaluated first and then the partial derivatives in $\mathbf{J}_{\hat{\boldsymbol{\theta}}_{i-1}}(t)$ are calculated numerically using the outputs from previous step. However, the entries of $\mathbf{J}_{\hat{\boldsymbol{\theta}}_{i-1}}(t)$ can actually be calculated using the outputs of the derivative system in one step by restacking each entry $\frac{\partial y_{s}^{(i)}(t)}{\partial \boldsymbol{\theta}}$ of $\mathcal{Y}_{\boldsymbol{\theta}}(t)$ as in (11) and evaluating the gradient using $\hat{\boldsymbol{\theta}}_{i-1}$.

With the approximation in (10), the likelihood function is proportional to

$$
\begin{aligned}
& p(\mathbf{y}(t) \mid \boldsymbol{\theta}) \propto \exp \{- \frac{1}{2 \sigma^{2}}\left[\mathbf{r}_{i-1}(t)-\mathbf{J}_{\hat{\boldsymbol{\theta}}_{i-1}}(t)\left(\boldsymbol{\theta}-\hat{\boldsymbol{\theta}}_{i-1}\right)\right]^{T} \\
&\left.\cdot\left[\mathbf{r}_{i-1}(t)-\mathbf{J}_{\hat{\boldsymbol{\theta}}_{i-1}}(t)\left(\boldsymbol{\theta}-\hat{\boldsymbol{\theta}}_{i-1}\right)\right]\right\},
\end{aligned}
$$

where $\mathbf{r}_{i-1}(t)=\mathbf{y}(t)-\mathbf{y}_{s}\left(t ; \hat{\boldsymbol{\theta}}_{i-1}\right)$. Assuming multiple independent measurements $\mathbf{Y}=\left[\mathbf{y}\left(t_{1}\right) \ldots \mathbf{y}\left(t_{N}\right)\right]$, the negative log-likelihood function will have the form:

$$
\begin{aligned}
&-\ln p(\mathbf{Y} \mid \boldsymbol{\theta}) \propto \sum_{j=1}^{N}\left\{\frac{1}{2 \sigma^{2}}\left[\mathbf{r}_{i-1}\left(t_{j}\right)-\mathbf{J}_{\hat{\boldsymbol{\theta}}_{i-1}}\left(t_{j}\right)\left(\boldsymbol{\theta}-\hat{\boldsymbol{\theta}}_{i-1}\right)\right]^{T}\right. \\
&\left.\cdot\left[\mathbf{r}_{i-1}\left(t_{j}\right)-\mathbf{J}_{\hat{\boldsymbol{\theta}}_{i-1}}\left(t_{j}\right)\left(\boldsymbol{\theta}-\hat{\boldsymbol{\theta}}_{i-1}\right)\right]\right\} .
\end{aligned}
$$

Since (13) is quadratic in $\boldsymbol{\theta}$, taking the derivative of (13) with respective to $\boldsymbol{\theta}$ and setting the result to zero leads to a closedform solution for $\boldsymbol{\theta}$, which suggests the following iterative ML estimator:

$$
\hat{\boldsymbol{\theta}}_{i}^{\mathrm{appML}}=\hat{\boldsymbol{\theta}}_{i-1}^{\mathrm{appML}}+\mathbf{M}_{A}^{-1} \mathbf{v}_{B},
$$

where

$$
\begin{aligned}
\mathbf{M}_{A} & =\frac{1}{\sigma^{2}} \sum_{j=1}^{N} \mathbf{J}_{\hat{\boldsymbol{\theta}}_{i-1}}^{T}\left(t_{j}\right) \mathbf{J}_{\hat{\boldsymbol{\theta}}_{i-1}}\left(t_{j}\right) \\
\mathbf{v}_{B} & =\frac{1}{\sigma^{2}} \sum_{j=1}^{N} \mathbf{J}_{\hat{\boldsymbol{\theta}}_{i-1}}^{T}\left(t_{j}\right) \mathbf{r}_{i-1}\left(t_{j}\right) .
\end{aligned}
$$

The "appML" superscript indicates that the estimate obtained from this iteration, which can only converge in general to a local minimum and is not based on the exact log-likelihood, will be an approximation to the ML solution.

In summary, the appML algorithm for estimating $\boldsymbol{\theta}$ consists of the following steps:

(1) Initialize $\hat{\boldsymbol{\theta}}_{0}$ and set $i=1$.

(2) Evaluate the derivative system to obtain $\mathbf{J}_{\hat{\boldsymbol{\theta}}_{i-1}}$.

(3) Use (14) to update the parameter estimate, and increment $i$.

(4) Repeat steps 2 and 3 until a convergence criterion is satisfied.

\section{B. Posterior Probability Function and MAP Estimator}

With the availability of a priori pdfs for the parameters, the MAP estimator maximizes the posterior $\mathrm{pdf}$

$$
p(\boldsymbol{\theta} \mid \mathbf{Y}) \propto p(\mathbf{Y} \mid \boldsymbol{\theta}) p(\boldsymbol{\theta})
$$

over $\boldsymbol{\theta}$. Since the DCM connection parameters are always positive, lognormal priors have typically been used to describe them [10]. Assuming independent parameters with lognormal priors, the joint prior pdf is then proportional to

$$
p(\boldsymbol{\theta}) \propto \prod_{k=1}^{n}\left\{\frac{1}{\theta_{k}} \exp \left[-\frac{\left(\ln \theta_{k}-\mu_{k}\right)^{2}}{2 \nu_{k}}\right]\right\},
$$


where $\mu_{k}$ and $\nu_{k}$ are the mean and variance for the pdf of the $k$ th parameter. Together with (13) and (15), this leads to the following expression for the negative log-probability function:

$$
\begin{gathered}
-\ln p\left(\boldsymbol{\theta} \mid \mathbf{Y} ; \hat{\boldsymbol{\theta}}_{i-1}\right) \propto \sum_{k=1}^{n}\left\{\ln \theta_{k}+\frac{\left(\ln \theta_{k}-\mu_{k}\right)^{2}}{2 \nu_{k}}\right\} \\
+\sum_{j=1}^{N}\left\{\frac{1}{2 \sigma^{2}}\left[\mathbf{r}_{i-1}\left(t_{j}\right)-\mathbf{J}_{\hat{\boldsymbol{\theta}}_{i-1}}\left(t_{j}\right)\left(\boldsymbol{\theta}-\hat{\boldsymbol{\theta}}_{i-1}\right)\right]^{T}\right. \\
\left.\cdot\left[\mathbf{r}_{i-1}\left(t_{j}\right)-\mathbf{J}_{\hat{\boldsymbol{\theta}}_{i-1}}\left(t_{j}\right)\left(\boldsymbol{\theta}-\hat{\boldsymbol{\theta}}_{i-1}\right)\right]\right\}
\end{gathered}
$$

Unlike the ML case, (17) is not quadratic in $\boldsymbol{\theta}$, and a closedform solution does not exist. A similar iterative technique can still be used to estimate the parameters:

$$
\hat{\boldsymbol{\theta}}_{i}^{\mathrm{appMAP}}=\arg \min _{\boldsymbol{\theta}}-\ln p\left(\boldsymbol{\theta} \mid \mathbf{Y} ; \hat{\boldsymbol{\theta}}_{i-1}^{\mathrm{appMAP}}\right)
$$

but a multidimensional optimization is required at each step rather than a simple matrix-vector multiply as in (14). Due to the use of the local linear approximation of $\mathbf{y}_{s}(t)$, this approach is referred to as the approximate MAP (appMAP) estimate. The appMAP algorithm is implemented in the same manner as appML, except that the parameters are updated using (18) in step (3).

Due to the overall complexity of the problem, a significant number of iterations is often required to reach convergence. Note that, although we have formulated the problem assuming that $\mathbf{C}_{\mathbf{w}}=\sigma^{2} \mathbf{I}$ is known, in principle it is possible to jointly estimate both the DCM parameters and the measurement noise covariance. In this case, the PCRB derivation of the previous section would have to be updated to include the unknown noise parameters.

\section{Dual Extended Kalman Filter}

A drawback of appML and appMAP is that when the parameters are updated, the DCM derivative system needs to be evaluated again before proceeding to the next iteration. Since convergence can take many iterations, implementation of the appML and appMAP is computationally demanding and time-consuming. Furthermore, the local linear approximation described above is in the parameter space, and if the initial guess for $\boldsymbol{\theta}$ is far from the true value, the approximation in (10) may not hold. In such cases, the algorithms will often converge to a local minimum far from the desired estimate. This can be true even if prior pdfs are available if (as discussed later in Section VI) both standard and rare stimuli are present. A prior pdf may be available for connectivity parameters under the standard stimulus, but often not for the case of a rare or oddball stimulus. An oddball event can lead to a large change in the connectivity parameters, and thus the algorithm initialized based on the prior can result in misconvergence.

In this section, we consider an alternative approach based on the use of the so-called dual extended Kalman filter (dEKF) [26]. While the dEKF does not lay claim to the statistical opti- mality enjoyed by MAP and ML (assuming they converge globally), it is much simpler to implement and is not sensitive to a poor parameter intialization. The computational benefit of the dEKF algorithm results from its ability to simultaneously estimate the states of the dynamical system and its parameters at each time instant, without the need for a derivative system implementation.

To begin with, consider a discrete-time version of the nonlinear system in (3) denoted by

$$
\begin{aligned}
\mathbf{x}_{k} & =\mathbf{F}\left(\mathbf{x}_{k-1}, \mathbf{u}_{k-1}, \boldsymbol{\theta}\right)+\boldsymbol{\xi}_{k} \\
\mathbf{y}_{k} & =\mathbf{C x}_{k}+\mathbf{w}_{k},
\end{aligned}
$$

where $\mathbf{x}_{k}, \mathbf{y}_{k}, \mathbf{u}_{k}$ and $\mathbf{w}_{k}$ are samples of $\mathbf{x}(t), \mathbf{y}(t), \mathbf{u}(t)$ and $\mathbf{w}(t)$ at time $t_{k}$. The vector $\boldsymbol{\xi}_{k}$ represents zero-mean normally distributed process noise with covariance $\mathbf{C}_{\boldsymbol{\xi}}$ used to model uncertainties in the system dynamics. While the model in (3) essentially assumes $\mathbf{C}_{\boldsymbol{\xi}}=\mathbf{0}$, we will retain $\mathbf{C}_{\boldsymbol{\xi}}$ in the equations that follow for completeness. The $\mathbf{F}\left(\mathbf{x}_{k-1}, \mathbf{u}_{k-1}, \boldsymbol{\theta}\right)$ term is obtained by discretizing the state equations in (3):

$$
\mathbf{F}\left(\mathbf{x}_{k-1}, \mathbf{u}_{k-1}, \boldsymbol{\theta}\right)=\mathbf{x}_{k-1}+\Delta t \mathbf{f}\left(\mathbf{x}_{k-1}, \mathbf{u}_{k-1}, \boldsymbol{\theta}\right)
$$

where $\Delta t$ is the sampling interval.

The basic idea of the dEKF is to jointly run two extended Kalman filters (EKFs), one for the state variables and one for the parameter estimates. The nonlinear system model is linearized in the vicinity of the previous state and parameter estimates, and then updated at each time index $k$. The time-update equations for the parameter filter are

$$
\begin{aligned}
\hat{\boldsymbol{\theta}}_{k}^{-} & =\hat{\boldsymbol{\theta}}_{k-1} \\
\mathbf{P}_{\boldsymbol{\theta}_{k}}^{-} & =\lambda^{-1} \mathbf{P}_{\boldsymbol{\theta}_{k-1}},
\end{aligned}
$$

where the forgetting factor $\lambda \in(0,1]$ provides an exponentially decaying weight on past data. The state filter equations are given by

$$
\begin{aligned}
\hat{\mathbf{x}}_{k}^{-} & =\mathbf{F}\left(\hat{\mathbf{x}}_{k-1}, \mathbf{u}_{k}, \hat{\boldsymbol{\theta}}_{k}^{-}\right) \\
\mathbf{P}_{\mathbf{x}_{k}}^{-} & =\mathbf{A}_{k-1} \mathbf{P}_{\mathbf{x}_{k-1}} \mathbf{A}_{k-1}^{T}+\mathbf{C}_{\boldsymbol{\xi}} .
\end{aligned}
$$

The measurement-update equations for the state filter are

$$
\begin{aligned}
\mathbf{K}_{k}^{\mathbf{x}} & =\mathbf{P}_{\mathbf{x}_{k}}^{-} \mathbf{C}^{T}\left(\mathbf{C} \mathbf{P}_{\mathbf{x}_{k}}^{-} \mathbf{C}^{T}+\sigma^{2} \mathbf{I}\right)^{-1} \\
\hat{\mathbf{x}}_{k} & =\hat{\mathbf{x}}_{k}^{-}+\mathbf{K}_{k}^{\mathbf{x}}\left(\mathbf{y}_{k}-\mathbf{C} \hat{\mathbf{x}}_{k}^{-}\right) \\
\mathbf{P}_{\mathbf{x}_{k}} & =\left(\mathbf{I}-\mathbf{K}_{k}^{\mathbf{x}} \mathbf{C}\right) \mathbf{P}_{\mathbf{x}_{k}}^{-},
\end{aligned}
$$

and those for the parameter filter are

$$
\begin{aligned}
\mathbf{K}_{k}^{\boldsymbol{\theta}} & =\mathbf{P}_{\boldsymbol{\theta}_{k}}^{-}\left(\mathbf{C}_{k}^{\boldsymbol{\theta}}\right)^{T}\left[\mathbf{C}_{k}^{\boldsymbol{\theta}} \mathbf{P}_{\boldsymbol{\theta}_{k}}^{-}\left(\mathbf{C}_{k}^{\boldsymbol{\theta}}\right)^{T}+0.5 \mathbf{I}\right]^{-1} \\
\hat{\boldsymbol{\theta}}_{k} & =\hat{\boldsymbol{\theta}}_{k}^{-}+\mathbf{K}_{k}^{\boldsymbol{\theta}}\left(\mathbf{y}_{k}-\mathbf{C}_{\hat{\mathbf{x}}_{k}^{-}}\right) \\
\mathbf{P}_{\boldsymbol{\theta}_{k}} & =\left(\mathbf{I}-\mathbf{K}_{k}^{\boldsymbol{\theta}} \mathbf{C}_{k}^{\boldsymbol{\theta}}\right) \mathbf{P}_{\boldsymbol{\theta}_{k}}^{-}
\end{aligned}
$$


where

$$
\begin{aligned}
\mathbf{A}_{k-1} & \left.\equiv \frac{\partial \mathbf{F}\left(\mathbf{x}, \mathbf{u}_{k-1}, \hat{\boldsymbol{\theta}}_{k}^{-}\right)}{\partial \mathbf{x}}\right|_{\hat{\mathbf{x}}_{k-1}} \\
\mathbf{C}_{k}^{\boldsymbol{\theta}} & \left.\equiv \mathbf{C} \frac{\partial \hat{\mathbf{x}}_{k}^{-}}{\partial \boldsymbol{\theta}}\right|_{\hat{\boldsymbol{\theta}}_{k}^{-}} .
\end{aligned}
$$

Substituting (21) into (32), $\mathbf{A}_{k-1}$ is calculated as

$$
\mathbf{A}_{k-1}=\mathbf{I}_{8 m}+\left.\Delta t \frac{\partial \mathbf{f}\left(\mathbf{x}, \mathbf{u}_{k-1}, \hat{\boldsymbol{\theta}}_{k}^{-}\right)}{\partial \mathbf{x}}\right|_{\hat{\mathbf{x}}_{k-1}},
$$

where the derivative on the right hand side is just the Jacobian matrix defined in (8). However, taking the derivative of the state filter equations with respect to the parameters in (33) leads to the computation of recurrent derivatives since $\hat{\mathbf{x}}_{k}$ is a function of $\hat{\mathbf{x}}_{k-1}$, and both are functions of $\boldsymbol{\theta}$. This leads to [26]:

$$
\begin{aligned}
\frac{\partial \hat{\mathbf{x}}_{k}^{-}}{\partial \boldsymbol{\theta}} & =\frac{\partial \mathbf{F}\left(\hat{\mathbf{x}}_{k-1}, \mathbf{u}_{k-1}, \boldsymbol{\theta}\right)}{\partial \hat{\mathbf{x}}_{k-1}} \frac{\partial \hat{\mathbf{x}}_{k-1}}{\partial \boldsymbol{\theta}}+\frac{\partial \mathbf{F}\left(\hat{\mathbf{x}}_{k-1}, \mathbf{u}_{k-1}, \boldsymbol{\theta}\right)}{\partial \boldsymbol{\theta}} \\
\frac{\partial \hat{\mathbf{x}}_{k-1}}{\partial \boldsymbol{\theta}} & =\left(\mathbf{I}-\mathbf{K}_{k-1}^{\mathbf{x}} \mathbf{C}\right) \frac{\partial \hat{\mathbf{x}}_{k-1}^{-}}{\partial \boldsymbol{\theta}}
\end{aligned}
$$

where $\frac{\partial \mathbf{F}\left(\hat{\mathbf{x}}_{k-1}, \mathbf{u}_{k-1}, \boldsymbol{\theta}\right)}{\partial \boldsymbol{\theta}}$ and $\frac{\partial \mathbf{F}\left(\hat{\mathbf{x}}_{k-1}, \mathbf{u}_{k-1}, \boldsymbol{\theta}\right)}{\partial \hat{\mathbf{x}}_{k-1}}$ are evaluated at $\hat{\boldsymbol{\theta}}_{k}^{-}=\hat{\boldsymbol{\theta}}_{k-1}$. Note that to simplify the algorithm, (35) is obtained assuming that $\mathbf{K}_{k}^{\mathbf{x}}$ is independent of $\boldsymbol{\theta}$.

The dEKF is implemented using the recursive equations defined above once the state and parameter vectors and their covariances are intialized. In the simulations conducted later, we used the following initial values:

(1) The elements of $\hat{\boldsymbol{\theta}}_{0}$ were set to the modes of the prior pdf for each parameter,

(2) $\hat{\mathbf{x}}_{0}=0$

(3) $\mathbf{P}_{\boldsymbol{\theta}_{0}}$ was calculated assuming that each entry in $\boldsymbol{\theta}$ is uniformly distributed between a prespecified upper and lower bound,

(4) $\mathbf{P}_{\mathbf{x}_{0}}=\epsilon \mathbf{I}$, with $\epsilon$ chosen to be a small number.

\section{Model Selection CRiteria}

In many brain studies where DCM is applied, knowledge of which connections between cortical regions are changed can be more important than the precise numerical values representing those connections. For example, under the auditory oddball paradigm, rare audio events produce ERPs with mismatch negativity (MMN), and this phenomenon is believed to be due to learning-related changes in the corticocortical connectivities [10], [27]. The bounds and algorithms presented earlier are applicable once a given DCM structure has been postulated, but in practice there are typically several competing models that must be evaluated in terms of how well they explain the measured ERPs. In this section, we briefly discuss the use of model-order selection criteria to determine which DCM structure best describes the measured data.

Given a set of $N$ observations and a family of models (i.e., a parameterized family of probability densities) without prior pdfs for the parameters, the model that best fits the data can be selected by minimizing Akaike's information criterion (AIC) [28], [29]:

$$
\mathrm{AIC}=-2 \ln p\left(\mathbf{Y} \mid \hat{\boldsymbol{\theta}}_{\mathrm{ML}}\right)+2 n,
$$

where the first term is the maximum of the likelihood function (i.e., the likelihood function evaluated at the ML estimate $\hat{\boldsymbol{\theta}}_{\mathrm{ML}}$ ), while the second term penalizes the model complexity, measured in terms of the number of free parameters $n$ on which the model is based. Alternatively, the Bayesian information criterion (BIC) or Schwarz criterion [29], [30] is derived using Bayesian arguments, and one selects the model that yields the maximum posterior probability. The formula for the BIC is similar to AIC, but with a stronger penalty for model complexity when $N$ is large:

$$
\mathrm{BIC}=-2 \ln p\left(\mathbf{Y} \mid \hat{\boldsymbol{\theta}}_{\mathrm{ML}}\right)+n \ln N .
$$

The BIC has the same form as the minimum description length (MDL) criterion [31].

When prior probabilities for the parameters are available, generalized model-order selection criteria based on the MAP estimator can be formulated as follows [32], [33]:

$$
\begin{aligned}
& \mathrm{gAIC}=-2 \ln p\left(\hat{\boldsymbol{\theta}}_{\mathrm{MAP}} \mid \mathbf{Y}\right)+2 n / N, \\
& \mathrm{gBIC}=-2 \ln p\left(\hat{\boldsymbol{\theta}}_{\mathrm{MAP}} \mid \mathbf{Y}\right)+n \ln N / N .
\end{aligned}
$$

In Section VI, we examine the performance of the various selection criteria presented above, in addition to comparisons of the ML, MAP and dEKF estimators against the appropriate Cramér-Rao bounds.

\section{Simulations AND Discussions}

\section{A. Simulation Setup}

In this section, we compare the performance of the dEKF and approximate ML and MAP algorithms described above against the CRB/PCRB using a plausible DCM network for the so-called auditory oddball paradigm. Under this paradigm, rare audio events produce ERPs with MMN, and this phenomenon is believed to be due to changes in corticocortical connectivities. To investigate the underlying mechanism of the MMN, a plausible DCM network motivated by electrophysiological and neuroimaging studies was suggested in [27], and is depicted in Fig. 2. This model is used to describe the generation of responses to both standard and oddball audio inputs. Five lumped neural sources are assumed in this model $(m=5)$, two associated with the left and right primary auditory cortices (A1), two with the left and right superior temporal gyrus (STG) and one for the right inferior frontal gyrus (IFG). The model only includes the right-hemisphere IFG since it is reported to consistently produce stronger responses than the left IFG [27]. The audio cortices A1 (regions 1 and 5) receive the external audio stimulus from the ear canal via connections with unknown strength $c_{1}, c_{5}$. In the most general setting with all possible connections active, the audio cortices are linked to their respective ipsilateral STG (regions 2 and 4) with distinct forward and backward connection parameters $a_{21}^{F}, a_{12}^{B}, a_{45}^{F}$, 


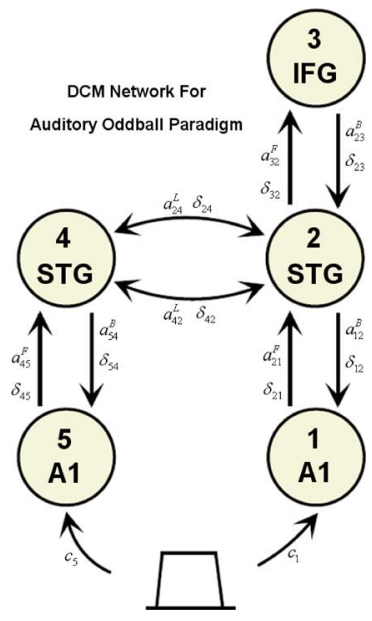

Fig. 2. A plausible DCM network for the auditory oddball paradigm.

$a_{54}^{B}$, and the conduction delays $\delta_{21}, \delta_{12}, \delta_{45}, \delta_{54}$ are assumed to be the same and equal to $\delta_{1}$ for both pathways. The right STG is connected to the IFG with parameters $a_{32}^{F}, a_{23}^{B}$ and conduction delay $\delta_{23}=\delta_{32}=\delta_{2}$. Inter-hemispheric or lateral connections between the left and right STG are defined by the parameters $a_{42}^{L}=a_{24}^{L}=a^{L}$ and $\delta_{24}=\delta_{42}=\delta_{3}$ (note that the forward and backward lateral connection strengths are assumed to be equal in the simulation since they exert much weaker effects on the system responses than forward and backward connections). Not all of the connection parameters will necessarily change under an oddball stimulus; we will use the model-order selection techniques presented earlier to test which connections are affected by the oddball event. When all of the connections are active, the parameter vector $\boldsymbol{\theta}$ is defined as

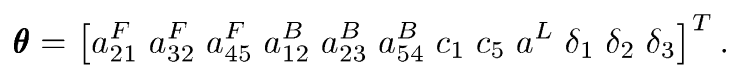

Lognormal priors denoted by $\ln \mathcal{N}(\mu, \nu)$ are assumed for the elements of $\boldsymbol{\theta}$ in [10], with mean and variance $\mu$ and $\nu$, respectively, as indicated in Table I. For a fair comparison, the MAP, ML and dEKF searches were all initialized using the modes of the prior distributions, and the searches were constrained over an interval surrounding the mode of each distribution. The modes as well as the upper and lower bounds for the numerical searches are also indicated in Table I. These bounds were chosen such that an integral of the prior pdf with these limits would yield $99.5 \%$ of the area under the pdf curve. Table II shows the value of the elements of $\boldsymbol{\theta}$ used in our simulation; the standard (non-oddball) values of the parameters were tuned so that the model generated ERP waveforms similar to those shown in [27]. The row labeled "Oddball Factor" contains a multiplier indicating the ratio of the parameter under the oddball paradigm to the parameter's standard value. These factors were also based on data in [27]. Note that under this model, only the connection parameters $a_{21}^{F}, a_{32}^{F}, a_{45}^{F}, a_{12}^{B}, a_{23}^{B}$ change as a result of the oddball audio input. The other DCM parameters were chosen to be similar to those used in prior work [9], [10], [15]: $H_{e}=3.5 \mathrm{mV}, H_{i}=32 \mathrm{mV}, \tau_{e}=10 \mathrm{~ms}$, $\tau_{i}=15 \mathrm{~ms}, \gamma_{1}=50, \gamma_{2}=40, \gamma_{3}=\gamma_{4}=12.5, e_{0}=2.5 \mathrm{~s}^{-1}$, $r=0.56 \mathrm{mV}^{-1}$. The input signal to the audio cortices was
TABLE I

Parameters of THE Prior Distributions For THE DCM NeTWORK

\begin{tabular}{|c|c|c|c|c|}
\hline Para. & Distribution & Mode & L Bound & U Bound \\
\hline \hline$c$ & $\ln \mathcal{N}(\ln 54,1 / 4)$ & 42.1 & 13.5 & 220 \\
$a^{F}$ & $\ln \mathcal{N}(\ln 32,1)$ & 11.8 & 2.0 & 530 \\
$a^{B}$ & $\ln \mathcal{N}(\ln 16,1)$ & 5.9 & 1.0 & 265 \\
$a^{L}$ & $\ln \mathcal{N}(\ln 4,1)$ & 1.5 & 0.25 & 66.25 \\
$\delta(\mathrm{ms})$ & $\ln \mathcal{N}(\ln 10,1 / 16)$ & 9.4 & 5.0 & 20.25 \\
\hline
\end{tabular}

TABLE II

Values of DCM Network Parameters Used in Simulating the AUDITORY ODDBALL PARADIGM

\begin{tabular}{|c|c|c|c|c|c|c|}
\hline Para. & $a_{21}^{F}$ & $a_{32}^{F}$ & $a_{45}^{F}$ & $a_{12}^{B}$ & $a_{23}^{B}$ & $a_{54}^{B}$ \\
\hline Standard & 40.56 & 61.42 & 31.75 & 8.67 & 13.81 & 8.81 \\
Oddball Factor & 0.31 & 6.05 & 2.04 & 3.08 & 1.55 & 1.0 \\
\hline \hline Para. & $c_{1}$ & $c_{5}$ & $a^{L}$ & $\delta_{1}$ & $\delta_{2}$ & $\delta_{3}$ \\
\hline Standard & 21.32 & 59.92 & 5.11 & 7.66 & 11.53 & 12.64 \\
Oddball Factor & 1.0 & 1.0 & 1.0 & 1.0 & 1.0 & 1.0 \\
\hline
\end{tabular}

a assumed to be a pulse with a $70 \mathrm{~ms}$ duration and $5 \mathrm{~ms}$ rise and fall times, as depicted in Fig. 2. After localization and interference suppression, the ERPs from each region were assumed to be reconstructed and sampled at $N$ time instants in the presence of spatially and temporally white noise $\left(\mathbf{C}_{\mathbf{w}}=\right.$ $\left.\sigma^{2} \mathbf{I}\right)$ at a given signal-to-noise ratio (SNR). The SNR is defined as $\left\|\mathbf{Y}_{s}\right\|_{F}^{2} /\|\mathbf{W}\|_{F}^{2}$, where $\mathbf{Y}_{s}=\left[\mathbf{y}_{s}(1), \ldots, \mathbf{y}_{s}(N)\right]$ and $\mathbf{W}=[\mathbf{w}(1), \ldots, \mathbf{w}(N)]$ represent the samples of the noise-free ERPs and the noise, respectively. The approximate ML and MAP iterations were run until the the sum of squared change in the parameter estimates between two iterations fell below $10^{-6}$ [6]. In the results that follow, 50 independent trials with different measurement noise realizations were conducted for each point on the plots. The DCM dynamic system and its derivative system were integrated using a fourth-order Runge-Kutta technique with an integration time-step specified by the sampling rate used with data collected during a 0.25 second time window.

\section{B. Estimation Results}

Fig. 3 shows the normalized root-mean-squared errors (RMSEs) and CRBs for estimation of the backward connection parameter, $a_{23}^{B}$, using dEKF, appML and appMAP. The simulations were conducted assuming a standard stimulus, a $1 \mathrm{kHz}$ sampling rate and the SNR was varied from 5 to 20 $\mathrm{dB}$. Red solid and dashed lines correspond to the CRB and PCRB, respectively. The symbols on each line correspond to the algorithm, with circles for $\mathrm{dEKF}$, squares for appML and diamonds for appMAP. Note that both appML and appMAP provide very similar performance essentially equivalent to the bounds. The benefit of the prior information is also apparent for $a_{23}^{B}$, and here appMAP has a clear advantage over appML, although both algorithms achieve their respective bounds. As expected, the performance of dEKF is somewhat worse than appML and appMAP, although these estimates are obtained with considerably less effort. Note that the RMSE of dEKF experiences a performance floor at high SNR due to the linearization of the model; this floor would be lowered and the performance of dEKF will improve if the sampling frequency is increased. 


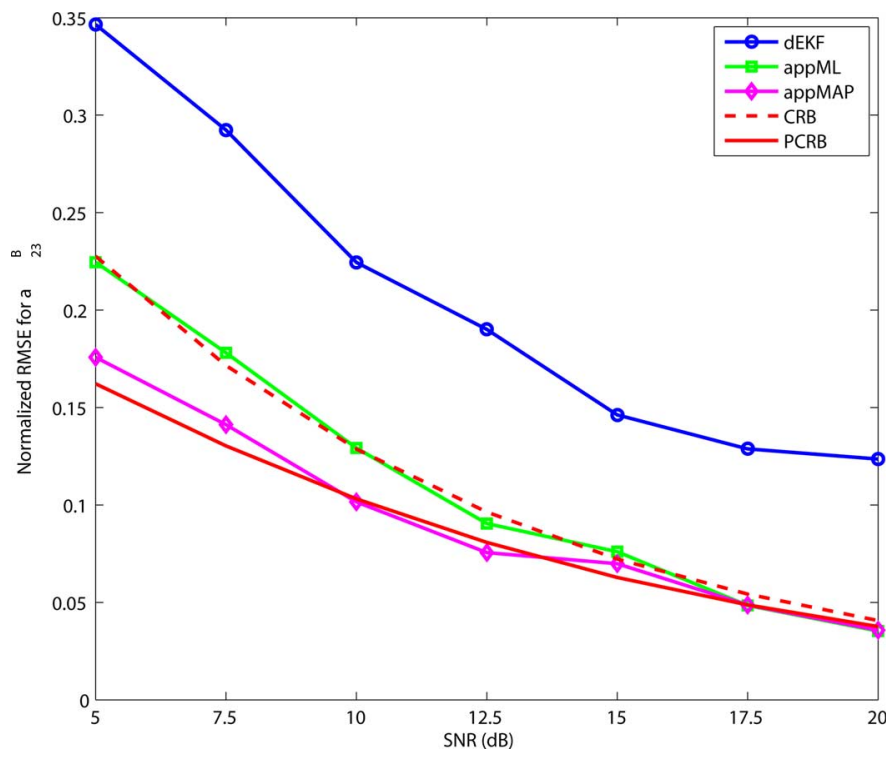

Fig. 3. Normalized RMSE for estimating the backward connection parameter, $a_{23}^{B}$, using dEKF, appML and appMAP. Normalized CRB (red dashed line) and normalized PCRB (red solid lines) are also depicted for comparison. Assumes standard stimulus and $1 \mathrm{kHz}$ sampling rate.

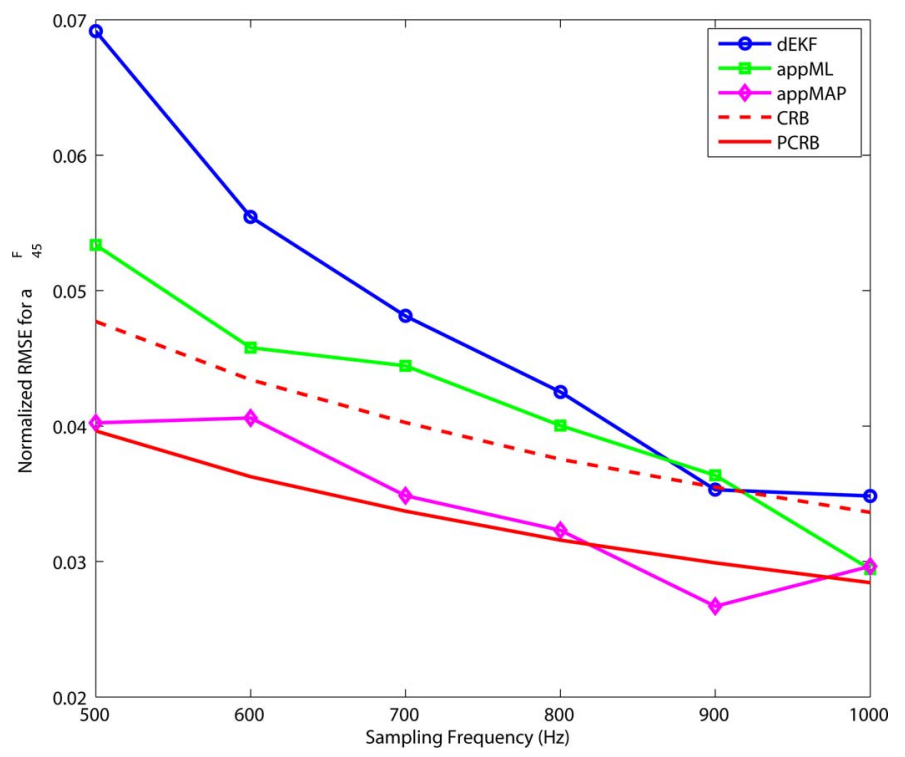

Fig. 4. Normalized RMSE for estimating the forward connection parameter $a_{45}^{F}$ using dEKF, appML and appMAP. Normalized CRB (red dashed line) and normalized PCRB (red solid lines) are also depicted for comparison. Under standard stimulus and SNR $=10 \mathrm{~dB}$.

In the second example, we illustrate the estimation performance for $a_{45}^{F}$ as the sampling frequency is varied from 500 to $1000 \mathrm{~Hz}$, again assuming a standard stimulus. The results are plotted in Fig. 4. In this case, the prior information also provides a visible improvement in RMSE for the parameter, and the appMAP approach outperforms appML as predicted by the bounds. The dEKF results show a more consistent improvement with increasing sampling rate than with SNR, due to the increased accuracy of the linearized approximations. To show this, in Fig. 5 we extend the previous example and plot the performance of dEKF as the sampling rate is increased to $5 \mathrm{kHz}$

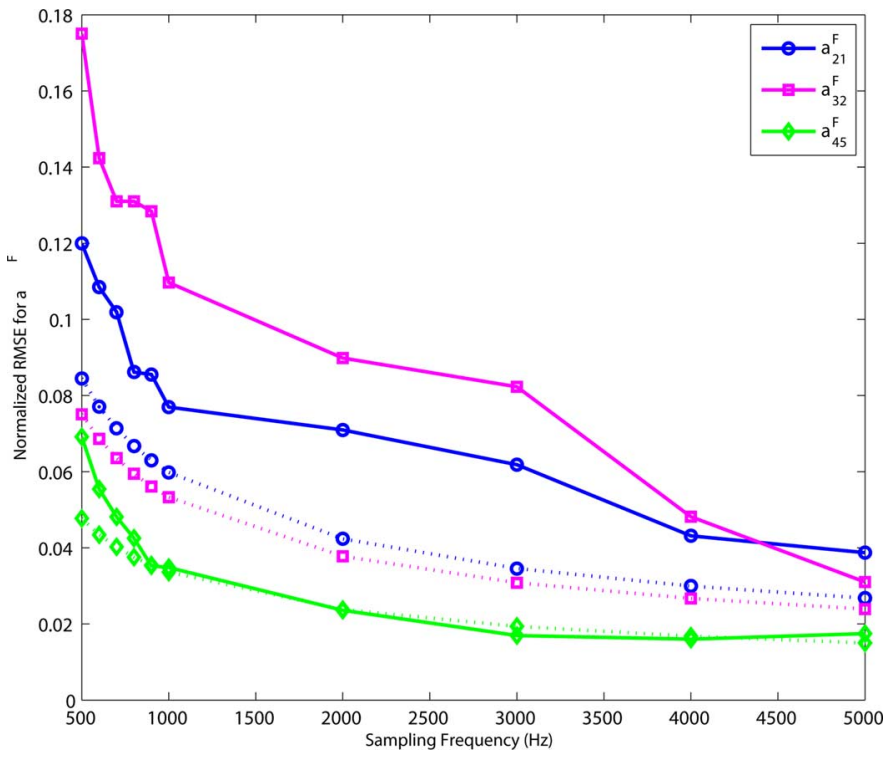

Fig. 5. Normalized RMSE and CRB for all three forward connection parameters estimation using dEKF. Same as Fig. 4 but sampling rate ranges from 500 $\mathrm{Hz}$ to $5 \mathrm{kHz}$.

for all three forward forward connection parameters, illustrating how the gap between the dEKF and the CRB shrinks at higher sampling rates.

\section{Model Order Selection Results}

We now explore the ability of the estimation algorithms to differentiate between changes in the DCM model under the assumption of an oddball stimulus, where MMN is observed in response to an unpredictable but recognizable audio event. In particular, it was assumed that the value of all parameters under the standard stimulus was known (e.g., from a previous estimation step), and it was desired to determine which parameters changed in response to the oddball stimulus. Three possible model changes were hypothesized, corresponding to whether the oddball event triggered changes in either the forward $(\mathrm{F})$, backward (B) or forward and backward (FB) connections. The parameters that are assumed to change under each hypothesis are given by

$$
\begin{aligned}
& \boldsymbol{\theta}_{F}=\left[\begin{array}{lll}
a_{21}^{F} & a_{32}^{F} & a_{45}^{F}
\end{array}\right] \\
& \boldsymbol{\theta}_{B}=\left[\begin{array}{lll}
a_{12}^{B} & a_{23}^{B} & a_{54}^{B}
\end{array}\right] \\
& \boldsymbol{\theta}_{F B}=\left[\begin{array}{llllll}
a_{21}^{F} & a_{32}^{F} & a_{45}^{F} & a_{12}^{B} & a_{23}^{B} & a_{54}^{B}
\end{array}\right] .
\end{aligned}
$$

Simulations were conducted with a $10 \mathrm{~dB}$ SNR and a sampling rate of $1 \mathrm{kHz}$, and oddball parameter values were chosen as given in Table II. The parameters that were assumed to remain unchanged under the oddball stimulus, namely $c_{1}, c_{5}, a^{L}$, and $\delta_{1}$ to $\delta_{3}$ (those with the oddball multipliers equal to 1.0 in Table II) were fixed at the values in the table and not estimated. Only the $a^{F}$ and $a^{B}$ parameters in (40)-(42) were estimated for each model, after which the model selection criteria were evaluated to determine which model best explained the observations. In addition to AIC and BIC, the value of the log-likelihood (or total probability) function without a penalty for model complexity was also tested as a model order selector. 


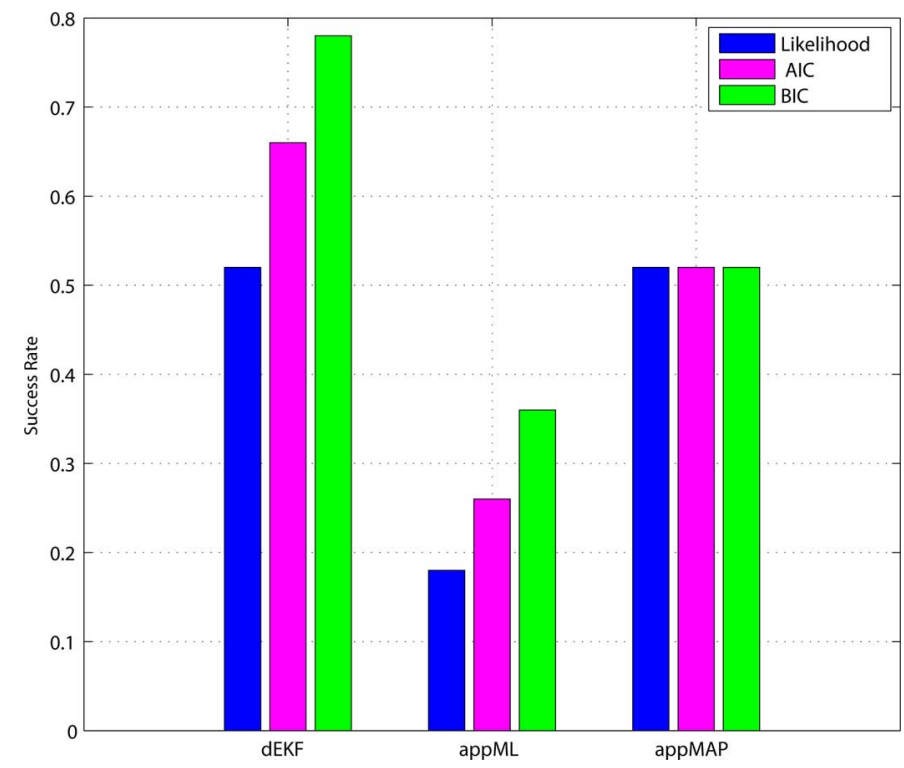

Fig. 6. Success rate of model selection based on three model selection criteria, in which parameter estimation was done using dEKF, appML or appMAP. The forward model was the actual underlying model.

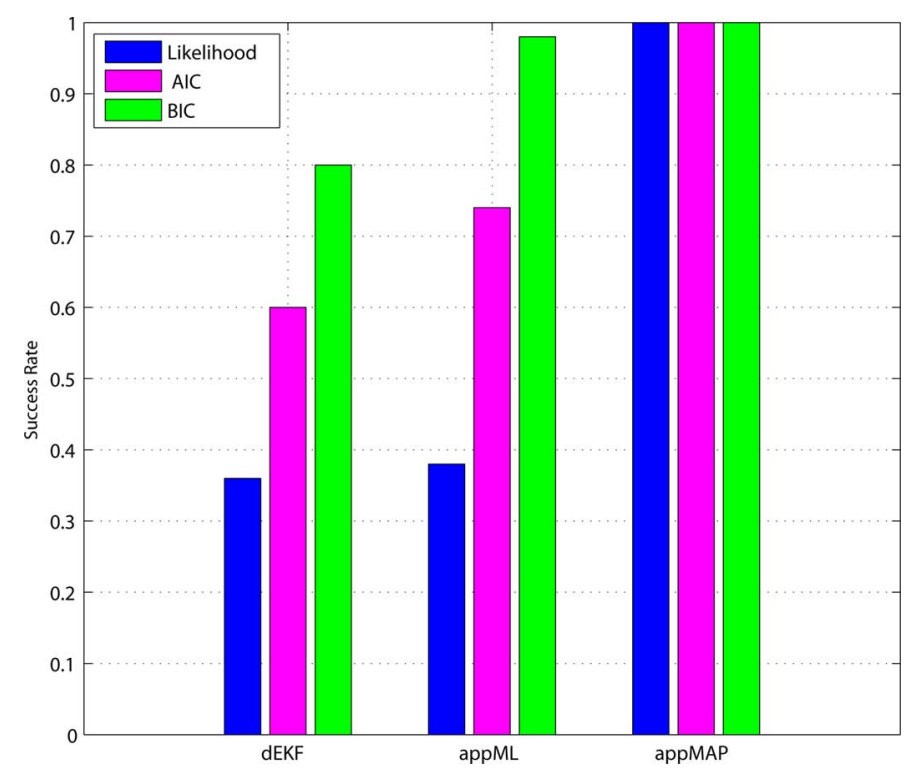

Fig. 7. Same as Fig. 6 but the backward model was the actual underlying model.

Fig. 6 shows the success rate of the three model selection approaches based on 50 different data realizations, with the forward $(F)$ hypothesis serving as the true underlying model change used to generate the data. Fig. 7 shows results for the case where the backward model was the actual underlying model. The performance of appML, appMAP and dEKF is shown for all three model selection criteria. Note that when appMAP was used as the estimator, the gAIC, gBIC and total probability functions were used rather than AIC, BIC and the log-likelihood. We clearly see the superiority of using BIC in all cases. The poor performance of the unpenalized likelihood function stems from the fact it often chooses the more complicated FB model in both cases, although we note that for the backward model, the appMAP estimation algorithm allows all three criteria to select the correct model in all 50 trials. The appMAP approach outperforms appML in these two examples, although interestingly the dEKF estimator results in a better success rate than appMAP for the forward model. This is likely due to the use of the local linear approximation together with initial values for the parameter search that are far removed from their actual values (due to the use of the oddball stimulus). The dEKF method appears to be more robust in this case, but it leads to worse performance for the backward model.

\section{CONCLUSION}

We have presented a nonlinear DCM framework for generation of EEG/MEG ERPs and applied this framework for investigating the mechanism of the MMN observed in the auditory oddball paradigm. Under the DCM framework, the MMN can be inferred as being due to changes in forward and backward connections within the primary auditory cortex compared to those present under standard stimuli. However, to make such an inference, the ability to estimate the DCM parameters is required, which results in a nonlinear system identification problem. We presented three algorithms to solve this problem, two based on the MAP and ML estimation paradigms, respectively (depending on whether or not prior information about the parameters is available), and a third based on the dual-EKF which is considerably simpler to implement. The MAP and ML approaches were based on local linear approximations to the cost functions, and as a result were referred to as appMAP and appML. The dEKF relies on linearizations in both the state and parameter space, and does not have a claim to statistical optimality. We also derived the CRB and posterior CRB for the DCM estimation problem, which requried the definition of the derivative system associated with the DCM since it is impossible to explicitly express the log-likelihood in terms of the model parameters. Our simulation results indicate that, despite the approximations, the appMAP and appML algorithms are able to achieve the PCRB and CRB, respectively, under the case of a standard stimulus, while the performance of dEKF is somewhat worse, although still reasonable. The use of the estimation algorithms in conjunction with selecting the DCM structure present under the oddball stimulus was also studied. The performance of the AIC and BIC model-order selection criteria was compared with the use of the likelihood function without a penalty for model complexity. The BIC approach provided the best performance in all cases examined, and appMAP outperformed appML due to its ability to exploit the prior parameter information. We also observed evidence that the dEKF approach may provide a degree of robustness under the oddball stimulus, when the parameters may be very different from their assumed a priori values.

\section{APPENDIX}

Derivatives for CALCUlating the $C R B$ : The Jacobian matrix of $\mathbf{f}$ with respect to $\mathbf{x}, \mathbf{M}_{J} \in \mathbb{R}^{8 m \times 8 m}$, is given by

$$
\mathbf{M}_{J}=\left[\left(\frac{\partial \mathbf{f}^{(1)}}{\partial \mathbf{x}}\right)^{T} \cdots\left(\frac{\partial \mathbf{f}^{(m)}}{\partial \mathbf{x}}\right)^{T}\right]^{T}
$$


where

$$
\begin{aligned}
& \frac{\partial \mathbf{f}^{(i)}}{\partial \mathbf{x}} \in \mathbb{R}^{8 \times 8 m} \\
& =\left[\begin{array}{ccccccc}
\frac{\partial f_{1}^{(i)}(t)}{\partial x_{1}^{(1)}} & \ldots & \frac{\partial f_{1}^{(i)}(t)}{\partial x_{8}^{(1)}} & \ldots & \frac{\partial f_{1}^{(i)}(t)}{\partial x_{1}^{(m)}} & \ldots & \frac{\partial f_{1}^{(i)}(t)}{\partial x_{8}^{(m)}} \\
\vdots & \ddots & \vdots & \ddots & \vdots & \ddots & \vdots \\
\frac{\partial f_{8}^{(i)}(t)}{\partial x_{1}^{(1)}} & \ldots & \frac{\partial f_{8}^{(i)}(t)}{\partial x_{8}^{(1)}} & \ldots & \frac{\partial f_{8}^{(i)}(t)}{\partial x_{1}^{(m)}} & \ldots & \frac{\partial f_{8}^{(i)}(t)}{\partial x_{8}^{(m)}}
\end{array}\right],
\end{aligned}
$$

in which most of the entries are zero except for the following:

$$
\begin{aligned}
& {\left[\mathbf{M}_{J}\right]_{(1,8(i-1)+4)}=1,\left[\mathbf{M}_{J}\right]_{(2,8(i-1)+5)}=1} \\
& {\left[\mathbf{M}_{J}\right]_{(3,8(i-1)+6)}=1,\left[\mathbf{M}_{J}\right]_{(4,8(i-1)+1)}=-\frac{1}{\tau_{e}^{2}}} \\
& {\left[\mathbf{M}_{J}\right]_{(4,8(i-1)+2)}=-\left[\mathbf{M}_{J}\right]_{(4,8(i-1)+3)}=\frac{H_{e}}{\tau_{e}} \gamma_{1} d S\left(y_{s}^{(i)}\right)} \\
& {\left[\mathbf{M}_{J}\right]_{(4,8(i-1)+4)}=-\frac{2}{\tau_{e}},\left[\mathbf{M}_{J}\right]_{(5,8(i-1)+1)}} \\
& =\frac{H_{e}}{\tau_{e}} \gamma_{2} d S\left(x_{1}^{(i)}\right) \\
& {\left[\mathbf{M}_{J}\right]_{(5,8(i-1)+2)}=-\frac{1}{\tau_{e}^{2}},\left[\mathbf{M}_{J}\right]_{(5,8(i-1)+5)}=-\frac{2}{\tau_{e}}} \\
& {\left[\mathbf{M}_{J}\right]_{(6,8(i-1)+3)}=-\frac{1}{\tau_{i}^{2}},\left[\mathbf{M}_{J}\right]_{(6,8(i-1)+6)}=-\frac{2}{\tau_{i}}} \\
& {\left[\mathbf{M}_{J}\right]_{(6,8(i-1)+7)}=\frac{H_{i}}{\tau_{i}} \gamma_{4} d S\left(x_{7}^{(i)}\right),\left[\mathbf{M}_{J}\right]_{(7,8(i-1)+8)}=1} \\
& {\left[\mathbf{M}_{J}\right]_{(8,8(i-1)+2)}=-\left[\mathbf{M}_{J}\right]_{(8,8(i-1)+3)}=\frac{H_{e}}{\tau_{e}} \gamma_{3} d S\left(y_{s}^{(i)}\right)} \\
& {\left[\mathbf{M}_{J}\right]_{(8,8(i-1)+7)}=-\frac{1}{\tau_{e}^{2}},\left[\mathbf{M}_{J}\right]_{(8,8(i-1)+8)}=-\frac{2}{\tau_{e}}} \\
& {\left[\mathbf{M}_{J}\right]_{(4,8(j-1)+2)}=\frac{H_{e}}{\tau_{e}}\left(a_{i j}^{F}+a_{i j}^{L}\right) d S\left(y_{s}^{(j)}\left(t-\delta_{i j}\right)\right)} \\
& {\left[\mathbf{M}_{J}\right]_{(4,8(j-1)+3)}=-\left[\mathbf{M}_{J}\right]_{(4,8(j-1)+2)}} \\
& {\left[\mathbf{M}_{J}\right]_{(5,8(j-1)+2)}=\frac{H_{e}}{\tau_{e}}\left(a_{i j}^{B}+a_{i j}^{L}\right) d S\left(y_{s}^{(j)}\left(t-\delta_{i j}\right)\right)} \\
& {\left[\mathbf{M}_{J}\right]_{(5,8(j-1)+3)}=-\left[\mathbf{M}_{J}\right]_{(5,8(j-1)+2)}} \\
& {\left[\mathbf{M}_{J}\right]_{(8,8(j-1)+2)}=\frac{H_{e}}{\tau_{e}}\left(a_{i j}^{B}+a_{i j}^{L}\right) d S\left(y_{s}^{(j)}\left(t-\delta_{i j}\right)\right)} \\
& {\left[\mathbf{M}_{J}\right]_{(8,8(j-1)+3)}=-\left[\mathbf{M}_{J}\right]_{(8,8(j-1)+2)},}
\end{aligned}
$$

where $i, j=1 \cdots m$ and $j \neq i$ and $d S(v)=\frac{d}{d v} S(v)$.

The derivatives of $\mathbf{f}$ with respect to $\theta$ can be represented as

$$
\frac{\partial \mathbf{f}}{\partial \theta}=\left[\left(\frac{\partial \mathbf{f}^{(1)}}{\partial \theta}\right)^{T} \cdots\left(\frac{\partial \mathbf{f}^{(m)}}{\partial \theta}\right)^{T}\right]^{T} \in \mathbb{R}^{8 m \times 1}
$$

where

$$
\frac{\partial \mathbf{f}^{(j)}}{\partial \theta}=\left[\begin{array}{c}
\frac{\partial f_{1}^{(j)}}{\partial \theta} \\
\vdots \\
\frac{\partial f_{8}^{(j)}}{\partial \theta}
\end{array}\right] \in \mathbb{R}^{8 \times 1}
$$

The non-zero entries of $\frac{\partial \mathbf{f}^{(j)}}{\partial \theta}$ are given by

$$
\begin{aligned}
& {\left[\frac{\partial \mathbf{f}^{(j)}}{\partial c_{i}}\right]_{4}=\frac{H_{e}}{\tau_{e}} u_{i}(t)} \\
& {\left[\frac{\partial \mathbf{f}^{(j)}}{\partial a_{j i}^{F}}\right]_{4}=\frac{H_{e}}{\tau_{e}} S\left(y_{s}^{(i)}\left(t-\delta_{j i}\right)\right)} \\
& {\left[\frac{\partial \mathbf{f}^{(j)}}{\partial a_{j i}^{B}}\right]_{5}=\left[\frac{\partial \mathbf{f}^{(j)}}{\partial a_{j i}^{B}}\right]_{8}=\frac{H_{e}}{\tau_{e}} S\left(y_{s}^{(i)}\left(t-\delta_{j i}\right)\right)}
\end{aligned}
$$$$
\left[\frac{\partial \mathbf{f}^{(j)}}{\partial a_{j i}^{L}}\right]_{4}=\left[\frac{\partial \mathbf{f}^{(j)}}{\partial a_{j i}^{B}}\right]_{5}=\left[\frac{\partial \mathbf{f}^{(j)}}{\partial a_{j i}^{B}}\right]_{8}=\frac{H_{e}}{\tau_{e}} S\left(y_{s}^{(i)}\left(t-\delta_{j i}\right)\right)
$$$$
\left[\frac{\partial \mathbf{f}^{(j)}}{\partial \delta_{j i}}\right]_{4}=-\frac{H_{e}}{\tau_{e}}\left(a_{j i}^{F}+a_{j i}^{L}\right) d S\left(y_{s}^{(i)}\left(t-\delta_{j i}\right)\right) \frac{d y_{s}^{(i)}\left(t-\delta_{j i}\right)}{d \delta_{j i}}
$$$$
\left[\frac{\partial \mathbf{f}^{(j)}}{\partial \delta_{j i}}\right]_{5}=-\frac{H_{e}}{\tau_{e}}\left(a_{j i}^{B}+a_{j i}^{L}\right) d S\left(y_{s}^{(i)}\left(t-\delta_{j i}\right)\right) \frac{d y_{s}^{(i)}\left(t-\delta_{j i}\right)}{d \delta_{j i}}
$$$$
\left[\frac{\partial \mathbf{f}^{(j)}}{\partial \delta_{j i}}\right]_{8}=-\frac{H_{e}}{\tau_{e}}\left(a_{j i}^{B}+a_{j i}^{L}\right) d S\left(y_{s}^{(i)}\left(t-\delta_{j i}\right)\right) \frac{d y_{s}^{(i)}\left(t-\delta_{j i}\right)}{d \delta_{j i}} .
$$

\section{REFERENCES}

[1] K. J. Friston, "Functional and effective connectivity in neuroimaging: A synthesis," Hum. Brain Mapp., vol. 2, pp. 56-78, 1994.

[2] L. Lee, L. M. Harrison, and A. Mechelli, "The functional brain connectivity workshop: Report and commentary," in NeuroImage, 2003, vol. 19 , pp. 457-465.

[3] B. Horwitz, "The elusive concept of brain connectivity," NeuroImage, vol. 19 , pp. 466-470, 2003

[4] A. R. Mcintosh and F. Gonzalez-Lima, "Structural equation modeling and its application to network analysis in functional brain imaging," Hum. Brain Mapp., vol. 2, pp. 2-22, 1994.

[5] M. Kaminski, M. Ding, W. A. Truccolo, and S. L. Bressler, "Evaluating causal relations in neural systems: Granger causality, directed transfer function and statistical assessment of significance," Biol Cybern., vol. 85, pp. 145-157, 2001.

[6] K. J. Friston, "Bayesian estimation of dynamical system: An application to fMRI," NeuroImage, vol. 16, pp. 513-530, 2002.

[7] L. Astolfi, F. Cincotti, C. Babiloni, F. Carducci, A. Basilisco, P. Rossini, S. Salinari, D. Mattia, S. Cerutti, D. Dayan, L. Ding, Y. Ni, B. He, and F. Babiloni, "Estimation of the cortical connectivity by high-resolution EEG and structural equation modeling: Simulations and application to finger tapping data," IEEE Trans. Biomed. Eng., vol. 52, pp. 757-768, 2005.

[8] L. Astolfi, F. Cincotti, D. Mattia, C. Babiloni, F. Carducci, A. Basilisco, P. M. Rossini, S. Salinari, L. Ding, Y. Ni, B. He, and F. Babiloni, “Assessing cortical functional connectivity by linear inverse estimation and directed transfer function: Simulations and application to real data," Clin. Neurophysiol., vol. 116, pp. 920-932, 2005.

[9] O. David, L. M. Harrison, and K. J. Friston, "Modelling event-related responses in the brain," NeuroImage, vol. 25, pp. 756-770, 2005.

[10] O. David, S. J. Kiebel, L. M. Harrison, J. Mattout, J. M. Kilner, and K. J. Friston, "Dynamic causal modeling of evoked responses in EEG and MEG," NeuroImage, vol. 30, pp. 1255-1272, 2006.

[11] L. Astolfi, F. Cincotti, D. Mattia, M. G. Marciani, L. A. Baccala, F. de Vico Fallani, S. Salinari, M. Ursino, M. Zavaglia, L. Ding, J. C. Edgar, G. A. Miller, B. He, and F. Babiloni, "Comparison of different cortical connectivity estimators for high-resolution EEG recordings," Hum. Brain Mapp., vol. 28, pp. 143-157, 2007.

[12] F. Darvas and R. M. Leahy, "Functional imaging of brain activity and connectivity with MEG," in Handbook of Brain Connectivity, V. K. Jirsa and A. R. McIntosh, Eds. New York, NY, USA: Springer, 2007, pp. 201-219.

[13] K. E. Stephan and K. J. Friston, "Models of effective connectivity in neural systems," in Handbook of Brain Connectivity, V. K. Jirsa and A. R. McIntosh, Eds. New York, NY, USA: Springer, 2007, pp. 303-327. 
[14] C. W. J. Granger, "Investigating causal relations by econometric models and cross-spectral methods," Econometrica, vol. 37, pp. 424-438, 1969.

[15] B. H. Jansen and V. G. Rit, "Electroencephalogram and visual evoked potential generation in a mathematical model of coupled cortical columns,” Biol. Cybern., vol. 73, pp. 357-366, 1995.

[16] O. David and K. J. Friston, "A neural mass model for EEG/MEG: Coupling and neuronal dynamics," NeuroImage, vol. 20, pp. 1743-1755, 2003.

[17] J. C. Mosher, P. S. Lewis, and R. M. Leahy, "Multiple dipole modeling and localization from spatio-temporal MEG data," IEEE Trans. Biomed. Eng., vol. 39, pp. 541-557, 1992.

[18] B. D. van Veen, W. van Drongelen, M. Yuchtman, and A. Suzuki, "Localization of brain electrical activity via linearly constrained minimum variance spatial filtering," IEEE Trans. Biomed. Eng., vol. 44, pp. 867-880, 1997.

[19] K. Sekihara, S. S. Nagarajan, D. Poeppel, A. Marantz, and Y. Miyashita, "Application of an MEG eigenspace beamformer to reconstructing spatio-temporal activities of neural sources," Hum. Brain Mapp., vol. 15, pp. 199-215, 2002.

[20] X.-L. Xu, B. Xu, and B. He, "An alternative subspace approach to EEG dipole source localization," Phys. Med. Biol., vol. 49, pp. 327-343, 2004.

[21] S. C. Wu, A. L. Swindlehurst, P. T. Wang, and Z. Nenadic, "Efficient dipole parameter estimation in EEG systems with near-ML performance," IEEE Trans Biomed Eng., vol. 59, pp. 1339-1348, 2012.

[22] S. J. Kiebel, O. David, and K. J. Friston, "Dynamic causal modelling of evoked responses in EEG/MEG with lead field parameterization," NeuroImage, vol. 30, pp. 1273-1284, 2006.

[23] S. M. Kay, Fundamentals of Statistical Signal Processing: Estimation Theory. Englewood Cliffs, NJ, USA: Prentice Hall, 1993.

[24] P. Tichavsky, C. H. Muravchik, and A. Nehorai, "Posterior Cramer-Rao bound for discrete-time nonlinear filtering," IEEE Trans. Signal Process., vol. 46, pp. 1386-1395, 1998.

[25] Z. Lin, Q. Zou, E. S. Ward, and R. J. Ober, "Cramer-Rao lower bound for parameter estimation in nonlinear systems," IEEE Signal Process. Lett., vol. 12, pp. 855-858, 2005.

[26] E. A. Wan and A. T. Nelson, "Dual extended Kalman filter methods," in Kalman Filtering and Neural Networks, S. Haykin, Ed. New York, NY, USA: Wiley, 2001, pp. 123-173.

[27] M. I. Garrido, J. M. Kilner, S. J. Kiebel, K. E. Stephan, and K. J. Friston, "Dynamic causal modelling of evoked potentials: A reproducibility study," NeuroImage, vol. 36, pp. 571-580, 2007.

[28] H. Akaike, "A new look at the statistical model identification," IEEE Trans. Autom. Control, vol. AC-19, pp. 716-723, 1974.

[29] W. D. Penny, K. E. Stephan, A. Mechelli, and K. J. Friston, "Comparing dynamic causal models," NeuroImage, vol. 22, pp. 1157-1172, 2004.

[30] G. Schwartz, "Estimating the dimension of a model," Ann. Stat., vol. 6, pp. 461-464, 1978

[31] J. Rissanen, "Modeling by shortest data description," Automatica, vol. 14, pp. 465-471, 1978.
[32] G. Sparacino, C. Tombolato, and C. Cobelli, "Maximum-likelihood versus maximum a posteriori parameter estimation of physiological system models-The C-peptide impulse response case study," IEEE Trans. Biomed. Eng., vol. 47, pp. 801-811, 2000.

[33] S. M. Veres, Structure Selection of Stochastic Dynamic Systems: The Information Criterion Approach. New York, NY, USA: Gordon and Breach, 1991.

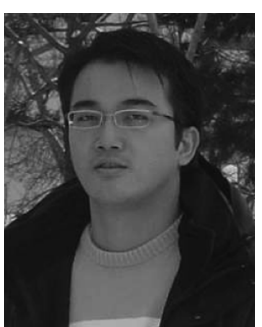

Shun Chi Wu (S'10-M'13) received the B.S. and M.S. degrees in engineering and system science from National Tsing Hua University, Hsinchu, Taiwan, in 2000 and 2002, respectively, and the Ph.D. degree in electrical engineering from University of California, Irvine, Irvine, California, in 2012.

From 2003 to 2007, he was a Research Assistant at National Space Organization, Hsinchu, Taiwan. His research interests include interference mitigation, feature extraction, source localization/reconstruction, and brain connectivity analysis.

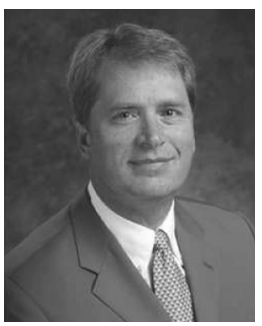

A. Lee Swindlehurst (S'83-M'84-SM'99-F'04) received the B.S., summa cum laude, and M.S. degrees in electrical engineering from Brigham Young University, Provo, UT, USA, in 1985 and 1986, respectively, and the Ph.D. degree in electrical engineering from Stanford University, Stanford, CA, USA, in 1991

From 1986 to 1990 , he was employed at ESL, Inc., of Sunnyvale, CA, where he was involved in the design of algorithms and architectures for several radar and sonar signal processing systems. He was on the faculty of the Department of Electrical and Computer Engineering at Brigham Young University from 1990 to 2007, where he was a Full Professor and served as Department Chair from 2003 to 2006. During 1996-1997, he held a joint appointment as a visiting scholar at both Uppsala University, Uppsala, Sweden, and at the Royal Institute of Technology, Stockholm, Sweden. From 2006 to 2007, he was on leave working as Vice President of Research for ArrayComm LLC in San Jose, CA. He is currently a Professor of Electrical Engineering and Computer Science at the University of California at Irvine. His research interests include sensor array signal processing for radar and wireless communications, detection and estimation theory, and system identification, and he has over 220 publications in these areas.

Dr. Swindlehurst is a past Secretary of the IEEE Signal Processing Society, past Editor-in-Chief of the IEEE Journal of SELECTED Topics IN SignaL PROCESSING, and past member of the Editorial Boards for the EURASIP Journal on Wireless Communications and Networking, IEEE Signal PRocessing Magazine, and the IEEE Transactions on Signal Processing. He is a recipient of several paper awards: the 2000 IEEE W. R. G. Baker Prize Paper Award, the 2006 and 2010 IEEE Signal Processing Society's Best Paper Award, the 2006 IEEE Communications Society Stephen O. Rice Prize in the Field of Communication Theory, and is co-author of a paper that received the IEEE Signal Processing Society Young Author Best Paper Award in 2001. 